\title{
Electrospun Nylon Fibres with Integrated Polypyrrole Molecularly Imprinted Polymers for the Detection of Glucose
}

\author{
Robert D. Crapnell ${ }^{1}$, Ryan J. Street ${ }^{1}$, Valentine Ferreira-Silva ${ }^{2}$, Michael P. Down ${ }^{1}$, Marloes Peeters ${ }^{3}$ \\ and Craig E. Banks*1
}

1) Faculty of Science and Engineering, Manchester Metropolitan University, John Dalton Building, Chester Street, Manchester, M1 5GD, United Kingdom.

2) Department of Mechanical Engineering, Institut National Des Sciences Appliquées Lyon, 69100 Villeurbanne, France.

3) School of Engineering, Newcastle University, Merz Court, Claremond Road, Newcastle Upon Tyne NE1 7RU, United Kingdom. 
Table S1. An short overview of various approaches reported towards the detection of glucose in sweat

\begin{tabular}{|c|c|c|c|c|c|}
\hline Sensing platform & Method of Detection & Linear Range & $\begin{array}{l}\text { Limit of } \\
\text { Detection }\end{array}$ & Sample Medium & Reference \\
\hline $\begin{array}{c}\mathrm{PVA} / \mathrm{BTCA} / \beta- \\
\mathrm{CD} / \mathrm{GOx} / \mathrm{AuNPs} \mathrm{NF} \\
\text { hydrogel }\end{array}$ & Amperometry & $0.1-0.5 \mathrm{mM}$ & $0.01 \mathrm{mM}$ & NR & 1 \\
\hline $\mathrm{ZnO} / \mathrm{PVP} \mathrm{NF}$ & Amperometry & 0.25 to $19 \mathrm{mM}$ & $1 \mu \mathrm{M}$ & NR & 2 \\
\hline $\begin{array}{l}\text { 2-hydroxy-1,4- } \\
\text { naphthoquinone }\end{array}$ & Optical & $1-250 \mathrm{mM}$ & NR & Artificial and human sweat & 3 \\
\hline $\mathrm{Cu}_{2} \mathrm{O} / \mathrm{Cu}$ & Amperometry & $0-2.5 \mathrm{mM}$ & $0.34 \mu \mathrm{M}$ & human sweat & 4 \\
\hline $\begin{array}{c}\mathrm{GOx} / \mathrm{PtNP} / \text { acetic acid } \\
\text { treated LIG }\end{array}$ & Amperometry & $0.0003-2.1 \mathrm{mM}$ & $1.3 \mu \mathrm{M}$ & human sweat & 5 \\
\hline PEDOT-NFs/GOx & Amperometry & $0.01-0.8 \mathrm{mM}$ & $165 \mu \mathrm{M}$ & Fruit juices & 6 \\
\hline $\mathrm{Cu}_{2} \mathrm{O} / \mathrm{GNs}$ & Amperometry & 0.3 to $7.8 \mathrm{mM}$ & $20.8 \mu \mathrm{M}$ & NR & 7 \\
\hline $\mathrm{CuO} / \mathrm{PANI}$ fibres & Amperometry & $0.005-9.255 \mathrm{mM}$ & $0.11 \mu \mathrm{M}$ & NR & 8 \\
\hline $\begin{array}{c}\text { Electrospun - } \\
\text { PMMA/Au/GOx }\end{array}$ & Amperometry & NR & $0.33 \mathrm{mM}$ & $\begin{array}{l}\text { Artificial sweat and serum } \\
\text { samples. }\end{array}$ & 9 \\
\hline $\begin{array}{l}\text { Electrospun Nylon-PPY- } \\
\text { MIPs }\end{array}$ & Thermal detection & $0.1-1 \mathrm{mM}$ & $0.1 \mathrm{mM}$ & Artificial sweat & This work \\
\hline
\end{tabular}

Key: NR: not reported; MIPs: molecular imprinted polymers; PPy: polypyrrole; Au: gold.; PMMA: poly(methyl methacrylate); GOx: Glucose oxidate.; PANI: Polyaniline; GNs: graphene nanosheets; PEDOT: Poly(3,4-ethylenedioxythiophene)); NFs: nanofibers; PtNP: platinum nanoparticles; LIG: laser induced graphene ; PVP: poly(vinyl pyrrolidone); PVA: poly(vinyl alcohol); BTCA: 1,2,3,4-butanetetracarboxylic acid (BTCA); $\beta$-CD: $\beta$-cyclodextrin polymer. 


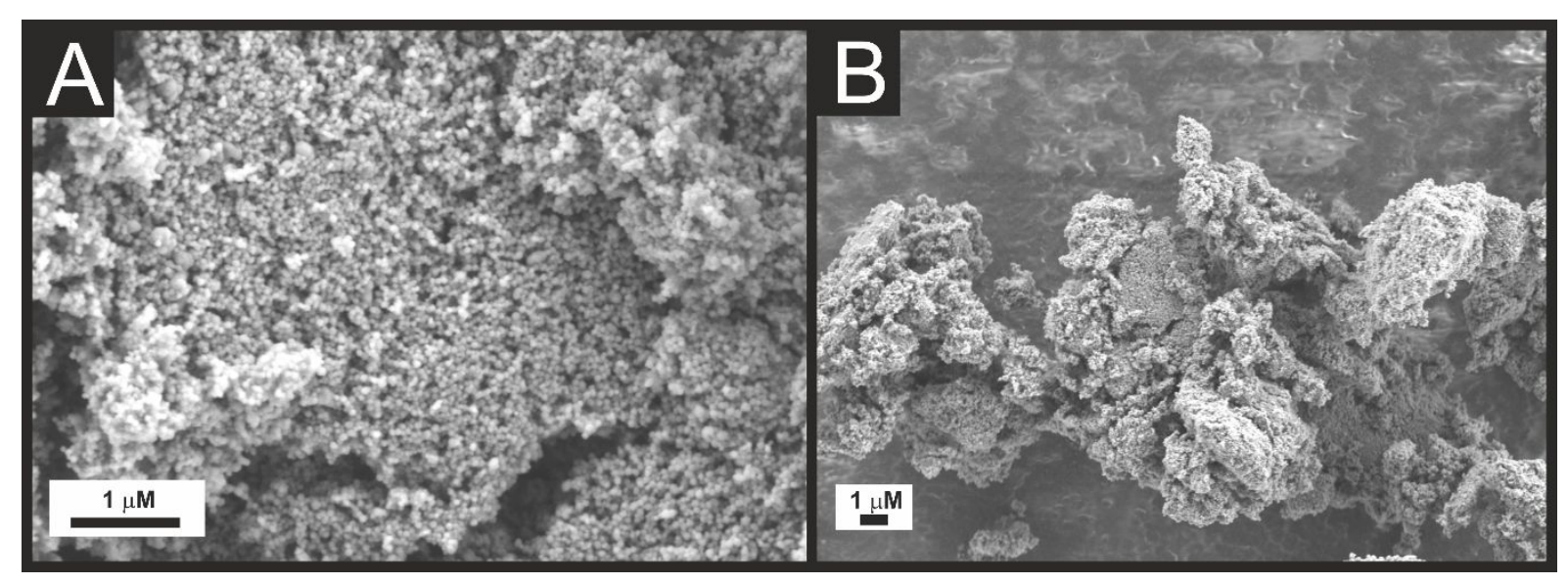

Figure S1. A) SEM image of PPy MIP powder formed at $40 \mathrm{k}$ magnification. B) SEM image of the PPy MIP powder formed at $5 \mathrm{k}$ magnification.

\section{References}

1. Kim, G. J.; Kim, K. O., Novel glucose-responsive of the transparent nanofiber hydrogel patches as a wearable biosensor via electrospinning. Sci. Rep. 2020, 10 (1), 18858.

2. Ahmad, M.; Pan, C.; Luo, Z.; Zhu, J., A Single ZnO Nanofiber-Based Highly Sensitive Amperometric Glucose Biosensor. J. Phys. Chem. C 2010, 114 (20), 9308-9313.

3. Mahmoud Al-omari; Gengchen Liu; Anja Mueller; Adam Mock; Ruby N. Ghosh; Kyle Smith; Tolga Kaya, A portable optical human sweat sensor. J. Appl. Phys. 2014, 116, , 183102.

4. Zhou, X.; Guo, W.; Yao, Y.; Peng, R.; Peng, P., Flexible Nonenzymatic Glucose Sensing with One-Step Laser-Fabricated Cu2O/Cu Porous Structure. Adv. Eng. Mater. 2021, 23, 2100192.

5. Yoon, H.; Nah, J.; Kim, H.; Ko, S.; Sharifuzzaman, M.; Barman, S. C.; Xuan, X.; Kim, J.; Park, J. Y., A chemically modified laser-induced porous graphene based flexible and ultrasensitive electrochemical biosensor for sweat glucose detection. Sens. Actuators, B 2020, 311, 127866.

6. Çetin, M. Z.; Camurlu, P., An amperometric glucose biosensor based on PEDOT nanofibers. RSC Adv. 2018, 8 (35), 19724-19731.

7. Liu, M.; Liu, R.; Chen, W., Graphene wrapped Cu2O nanocubes: Non-enzymatic electrochemical sensors for the detection of glucose and hydrogen peroxide with enhanced stability. Biosens. Bioelectron. 2013, 45, 206-212.

8. Liu, T.; Guo, Y.; Zhang, Z.; Miao, Z.; Zhang, X.; Su, Z., Fabrication of hollow CuO/PANI hybrid nanofibers for non-enzymatic electrochemical detection of $\mathrm{H} 2 \mathrm{O} 2$ and glucose. Sens. Actuators $B$ 2019, 286, 370-376.

9. $\quad$ Aldea, A.; Leote, R. J. B.; Matei, E.; Evanghelidis, A.; Enculescu, I.; Diculescu, V. C., Gold coated electrospun polymeric fibres as new electrode platform for glucose oxidase immobilization. Microchem. J. 2021, 165, 106108. 